\title{
Recent Advances in Designing 5-Fluorouracil Delivery Systems: A Stepping Stone in the Safe Treatment of Colorectal Cancer
}

This article was published in the following Dove Press journal: International Journal of Nanomedicine

\author{
Elaheh Entezar-Almahdi $\mathbb{D}^{\prime}$ \\ Soliman Mohammadi- \\ Samani (iD) ${ }^{1,2}$ \\ Lobat Tayebi iD ${ }^{3}$ \\ Fatemeh Farjadian (D) ${ }^{2}$ \\ 'Department of Pharmaceutics, Faculty of \\ Pharmacy, Shiraz University of Medical \\ Sciences, Shiraz, Iran; ${ }^{2}$ Pharmaceutical \\ Sciences Research Center, Shiraz \\ University of Medical Sciences, Shiraz, \\ Iran; ${ }^{3}$ Marquette University, School of \\ Dentistry, Milwaukee, WI, USA
}

Correspondence: Fatemeh Farjadian Pharmaceutical Sciences Research Center, Shiraz University of Medical Sciences, Shiraz 7|348-14336, Iran

Tel +987I32424I $27(302)$

Email farjadian_f@sums.ac.ir

\begin{abstract}
Fluorouracil (5-FU) has become one of the most widely employed antimetabolite chemotherapeutic agents in recent decades. It is considered a first line antineoplastic agent for the treatment of colorectal cancer. Unfortunately, chemotherapy with 5-FU has several limitations, including its short half-life, high cytotoxicity and low bioavailability. In order to overcome the drawbacks of 5-FU and enhance its therapeutic efficiency, many scientific groups have focused on designing a new delivery system to successfully deliver 5FU to tumor sites. We provide a comprehensive review on different strategies to design effective delivery systems, including nanoformulations, drug-conjugate formulations and other strategies for the delivery of 5-FU to colorectal cancer. Furthermore, co-delivery of 5-FU with other therapeutics is discussed. This review critically highlights the recent innovations in and literature on various types of carrier system for 5-FU.
\end{abstract}

Keywords: 5-fluorouracil, drug delivery, colorectal cancer, nanomedicine, nanoparticles, codelivery

\section{Introduction}

Colorectal cancer (CRC) is the third most prevalent cancer in both men and women, and the second for cancer-related mortality, following lung cancer. Various types of medicine or medicine combinations have been investigated as efficient treatments. ${ }^{1,2}$ Nanomedicine offers noteworthy solutions and is considered as a magic bullet for drug delivery in order to achieve maximum benefit, while minimizing side effects and improving drug efficacy. ${ }^{3-6}$ Smart nanoparticulated drug delivery systems (DDSs) (Figure 1) have made a great impact on the safe accumulation of anticancer drugs to the designated region and the delivery of therapeutic agents based on external or internal stimuli. ${ }^{5,7,8}$

5-Fluorouracil (5-FU) has become one of the most widely employed antimetabolite chemotherapeutic agents in recent decades. It has been used as a first line antineoplastic agent in the treatment of several cancers, such as colorectal, breast, head and neck, pancreas and stomach cancers. 5-FU is a water-soluble drug; hence, it is administered intravenously. 9,10 However, the appearance of drug resistance is substantial limitation in the clinical usage of 5-FU. For instance, owing to its low bioavailability, the cure rates of 5-FU for advanced CRC are less than $15 \%$. Moreover, 5-FU results in severe toxicological damage to the gastrointestinal (GI) system and blood factors; and neurological, dermatological and cardiological 


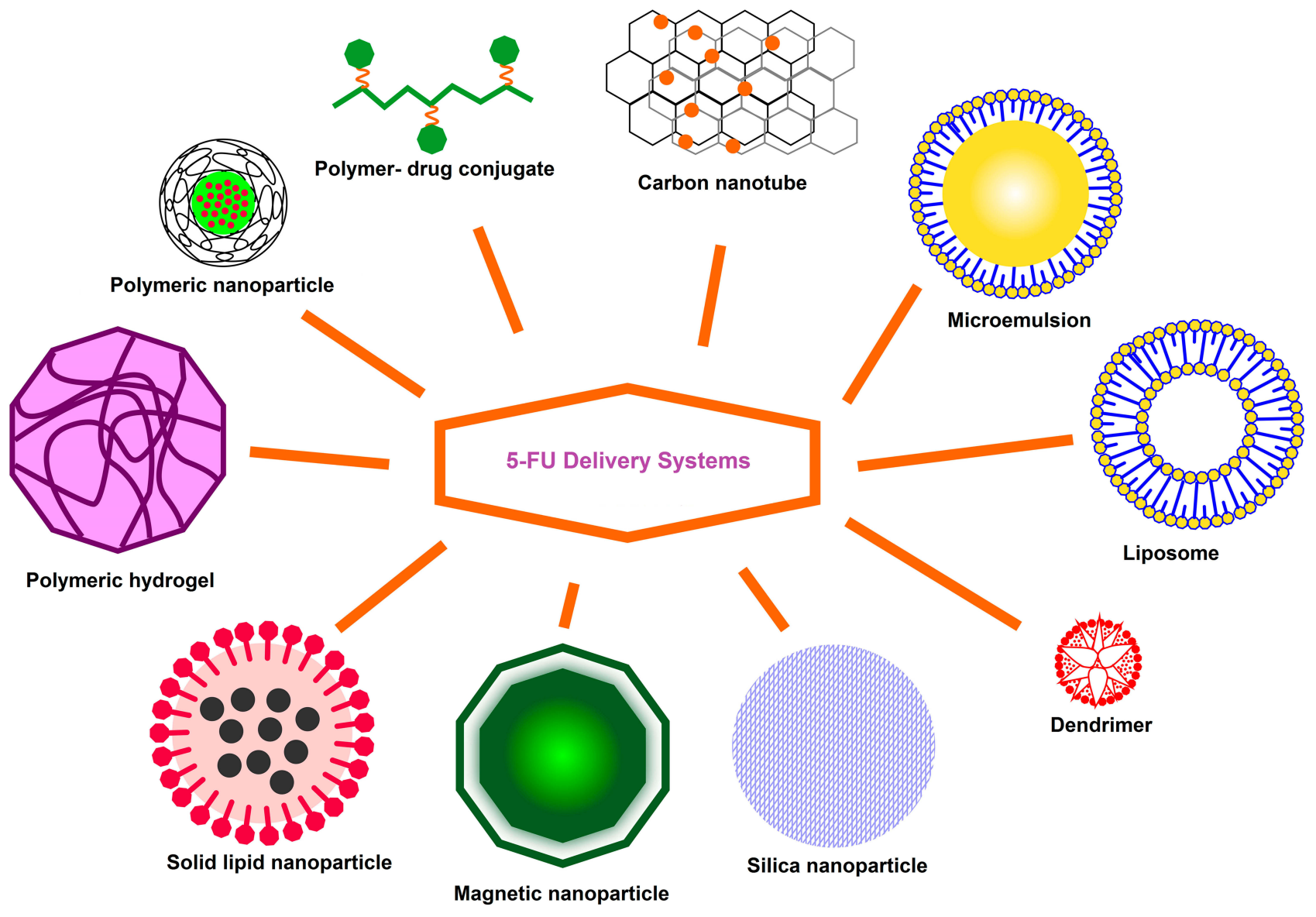

Figure I Some nanoparticulate DDSs.

reactions. ${ }^{5,11}$ Thus, it is essential to develop promising DDSs for 5-FU to achieve a better therapeutic effect with fewer side effects. Another strategy to achieve good results in the treatment of CRC is using fluorouracil substitutes such as TAS-102, which can overcome the short half-life and poor efficacy of 5-FU and have a good targeting effect. ${ }^{12}$

Although 5-FU is among the superior chemotherapeutic agents for CRC, there are several disadvantages, including rapid metabolism, short half-life (around 10-20 minutes), ${ }^{13}$ low bioavailability, high cell toxicity and inadequate selectivity for cancerous cells, all of which limit the effectiveness of 5-FU in cancer chemotherapy. Furthermore, oral formulations would result in seriously adverse GI reactions. In order to surmount the disadvantages of 5-FU and enhance its chemotherapeutic efficacy in CRC, many research teams have focused on the development of new 5-FU delivery systems. With this method, the cytotoxic effect of anticancer agents on healthy tissues can be lessened by using specific DDSs ${ }^{7,14,15}$
In this article, a comprehensive review of the literature, considering contemporary articles on DDSs for 5-FU, is presented. The mechanism of action and resistance to 5$\mathrm{FU}$ are also reviewed. Furthermore, potential strategies to design novel DDSs for 5-FU are discussed. Finally, we provide future outlooks on utilizing DDSs for 5-FU delivery to CRC.

\section{Mechanism of Action and Resistance to 5-FU}

As an analogue of the nucleobase uracil, 5-FU can enter cells via the same mechanism of facilitated transport as uracil. ${ }^{16}$ Then, 5-FU is converted to fluorodeoxyuridine monophosphate (FdUMP), fluorodeoxyuridine triphosphate (FdUTP) and fluorouridine triphosphate (FUTP), which are the active metabolites of 5-FU. RNA synthesis and the operation of thymidylate synthase (TS) are interrupted by these metabolites (Figure 2). ${ }^{9}$ By this action, 5FU can fight cancerous cells. 

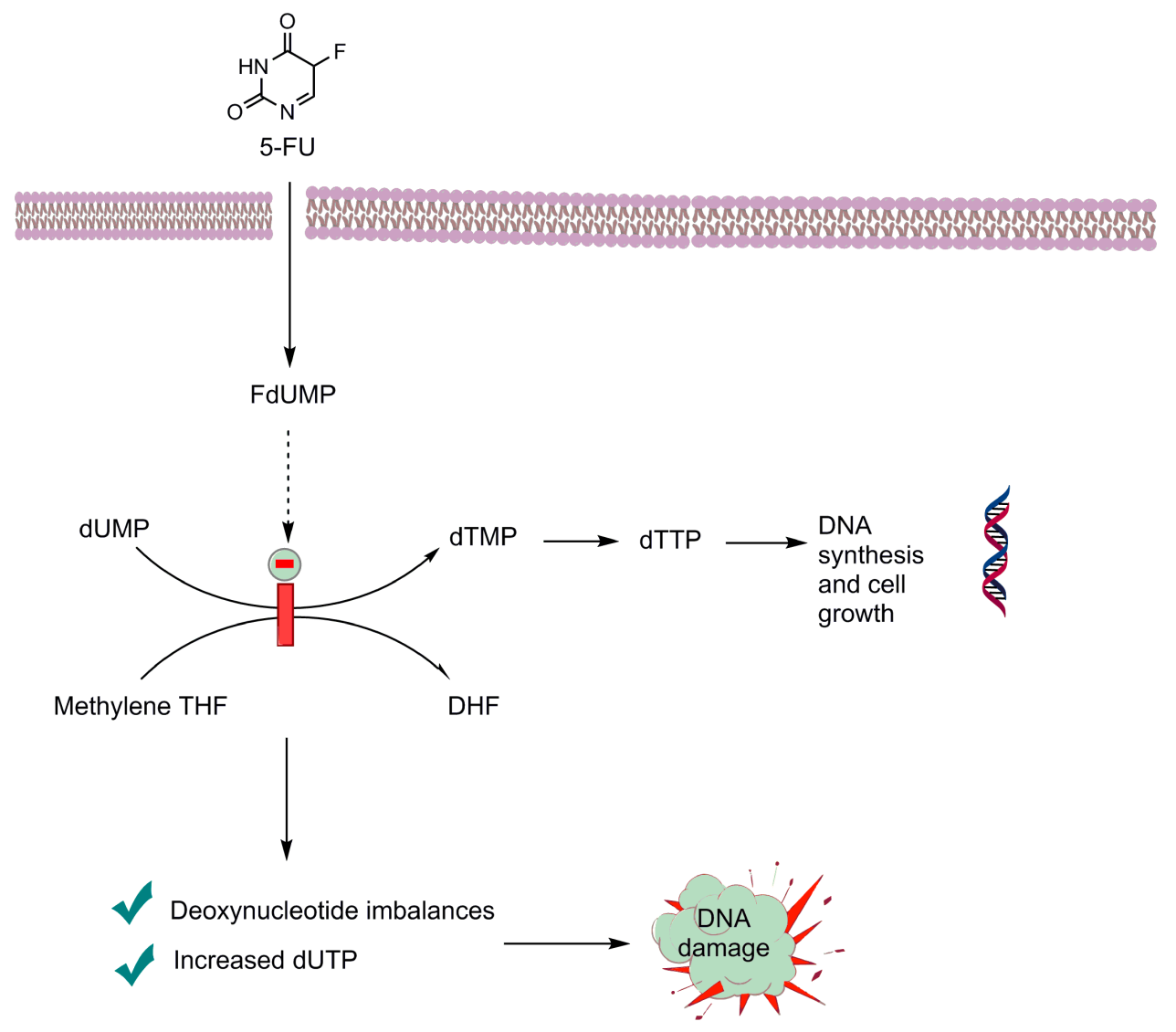

Figure 2 Mechanism of action of 5-FU to inhibit thymidylate synthase.

Abbreviations: FdUMP, fluorodeoxyuridine monophosphate; dUTP, deoxyuridine triphosphate; dTMP, deoxythymidine monophosphate; dTTP, deoxythymidine triphosphate methylene; THF, methylene tetrahydrofolate; DHF, dihydrofolate.

On the other hand, most of the provided 5-FU is catabolized to dihydrofluorouracil (DHFU), which is an inactive metabolite, by dihydropyrimidine dehydrogenase (DPD). This is a rate-limiting enzyme for 5-FU catabolism and is found to a great extent in liver and cancer cells. Upregulation of DPD gene expression in CRC is associated with 5-FU resistance. ${ }^{17}$ Therefore, higher doses of 5-FU are needed in CRC with acquired drug resistance (ADR). ${ }^{18}$

\section{Potential Strategies to Enhance Chemotherapy by 5-FU}

One of the most important purposes of pharmaceutical research is to discover and engineer new materials for formulating 5-FU, in order to minimize side effects but maximize clinical efficacy. ${ }^{19,20}$ Different methods can be used to construct drug carriers. For example, the use of a core-shell structure, where the specific drug is in the core of the DDS, can efficiently protect the drug from the environment and prolong its circulation time. ${ }^{21}$ This method results in physical entrapment of drug in the nanoformulation. In addition, using this strategy, passive accumulation of the DDS in the tumor environment can be achieved, which is called the enhanced permeability and retention effect. ${ }^{22,23}$ Furthermore, targeted delivery of the drug can be achieved by several receptors overexpressed on some tumor cells, such as asialoglycoproteins, ${ }^{24}$ transferrin, ${ }^{25}$ integrin receptors ${ }^{26,27}$ and folate. ${ }^{28}$

Another strategy used to make drug carrier systems involves chemically binding the drug molecule to a polymer chain to create a polymer-drug conjugate. In this way, burst release from the DDS is inhibited. This method has advantages in increasing the solubility of the drug and tunability of drug pharmacokinetics. ${ }^{22}$

In this section, we discuss three different types of delivery system used in pharmaceutical research to deliver 5-FU successfully. First, nanotechnology-based 5-FU delivery systems (Table 1) are discussed. Second, microparticulate delivery systems, and third, drug-conjugate delivery of 5-FU are considered and discussed. 
Table I Different Nano-Delivery Systems of 5-FU

\begin{tabular}{|c|c|c|c|}
\hline Main Format & Structure & Particle Size & Reference \\
\hline Solid Lipid Nanoparticle & Stearic acid, lecithin, poloxamer 188 & $\begin{array}{l}137 \pm 5.5 \mathrm{~nm} \text { to } 800 \pm 53.6 \\
\mathrm{~nm}\end{array}$ & 32 \\
\hline Chitosan & $\begin{array}{l}\text { CS-g-PCL } \\
\text { LMWC-4-bromomethyl-3-nitrobenzoic acid } \\
\text { CS-PAsp } \\
\text { CG5-FU-NPs and FCG5-FU-NPs }\end{array}$ & $\begin{array}{l}61.4-108.6 \mathrm{~nm} \\
365 \mathrm{~nm} \\
85-300 \mathrm{~nm} \\
31-33 \mathrm{~nm}\end{array}$ & $\begin{array}{l}33 \\
34 \\
35 \\
36\end{array}$ \\
\hline Magnetic Nanocarrier & $\begin{array}{l}\mathrm{Fe}_{3} \mathrm{O}_{4} \text {-encapsulating carbon nanospheres (TMMIPs) } \\
\text { Samarium ferrite }\left(\mathrm{SmFeO}_{3}\right) \text { nanoparticles coated with poly } \\
\text { (methylmethacrylate) } \\
\mathrm{CEINs}-\mathrm{PEI}-\beta \mathrm{CD}-\mathrm{FA}\end{array}$ & $\begin{array}{l}150 \mathrm{~nm} \\
\sim 50 \mathrm{~nm} \\
<100 \mathrm{~nm}\end{array}$ & $\begin{array}{l}40 \\
48 \\
49\end{array}$ \\
\hline $\begin{array}{l}\text { Molecularly Imprinted } \\
\text { Polymer }\end{array}$ & $\begin{array}{l}\text { MIP-CS-g-PMMA } \\
\text { TMMIPs }\end{array}$ & $\begin{array}{l}130 \mathrm{~nm} \\
150 \mathrm{~nm}\end{array}$ & $\begin{array}{l}39 \\
40\end{array}$ \\
\hline Polymeric NP and MP & $\begin{array}{l}\text { Poly(3-hydroxybutyrate-co-3 hydroxyhexanoate) (PHBHHx) } \\
\text { PLA and PLA-PEG } \\
\text { FA-PLGA }\end{array}$ & $\begin{array}{l}160 \mathrm{~nm} \text { and } 3 \mu \mathrm{m} \\
294 \mathrm{~nm} \text { and } 283 \mathrm{~nm} \\
224 \pm 18 \mathrm{~nm}\end{array}$ & $\begin{array}{l}41 \\
47 \\
42\end{array}$ \\
\hline Protein NP & Bovine serum albumin (BSA) & $210 \mathrm{~nm}$ & 43 \\
\hline Dendrimer & $\begin{array}{l}\text { PEG-PAMAM } \\
\text { PDEA-mPEG-PAMAM (PPD) }\end{array}$ & $\begin{array}{l}270-307 \mathrm{~nm} \\
43.0 \mathrm{~nm} \text { at } \mathrm{pH} 4.0 \\
41.6 \mathrm{~nm} \text { at } \mathrm{pH} 6.5 \text { and } \\
\mathrm{I} 1.6 \mathrm{~nm} \text { at } \mathrm{pH} 7.4\end{array}$ & $\begin{array}{l}45 \\
46\end{array}$ \\
\hline Liposome & $\begin{array}{l}\text { Transferrin-conjugated liposome } \\
\text { Folate-PEG-DSPE }\end{array}$ & $\begin{array}{l}- \\
\mathrm{I} 74 \mathrm{~nm}\end{array}$ & 52 \\
\hline $\begin{array}{l}\text { Carbon Nanotube } \\
\text { Silica NP }\end{array}$ & $\begin{array}{l}\text { Multi-walled CNTs } \\
\text { Epidermal growth factor-hollow mesoporous silica nanoparticles }\end{array}$ & $\begin{array}{l}- \\
\text { I } 20 \mathrm{~nm} \text { and } \\
\text { pore size }=2.5 \mathrm{~nm}\end{array}$ & $\begin{array}{l}56 \\
18\end{array}$ \\
\hline
\end{tabular}

\section{Recruitment of Nano-Based Delivery Systems}

\section{SLN-Based Delivery Systems}

Solid lipid nanoparticles (SLNs), as a colloidal DDS, have many advantages, including the ability to control the drug release pattern and enhance drug stability, and possess a high drug loading capacity. ${ }^{29-31}$

The incorporation of 5-FU into the shell of SLNs for the treatment of Erlich's tumor was investigated. The in vitro release behavior of prepared SLNs was improved in comparison with 5-FU alone. Moreover, the sodium carboxymethyl cellulose $(\mathrm{NaCMC})$ gel matrix was used as a suitable medium for SLN diffusion and release. SLN-treated mice exhibited improved treatment efficacy in comparison to 5-FU-injected mice. As a result, SLNs could enhance 5-FU or any other hydrophilic medicine through the local route of administration. ${ }^{32}$

\section{Chitosan as a Polymeric Nanocarrier CS-g-PCL}

In one study, a series of chitosan-graft-poly( $\varepsilon$-caprolactone) micelles (CS-g-PCL) for 5-FU delivery was prepared. It was found that the properties of the micelles were highly influenced by the length of the hydrophobic chain of the copolymer. These copolymers showed controlled release behavior and interaction levels between copolymers, and 5-FU was a determinant factor in tuning the release rate. 5-FU-loaded micelles displayed adequate biocompatibility and lower cytotoxicity in comparison to free 5-FU as a result of the slow release behavior, yet they were efficient in killing cancerous cells. ${ }^{33}$

\section{Low Molecular Weight Chitosan-5-FU Conjugate}

A prodrug of 5-FU covalently conjugated to low molecular weight chitosan (LMWC) with a photo-responsive linker 
(attachment of 5-FU with 4-bromomethyl-3-nitrobenzoic acid, which is the cleavable linker) was also reported. The linker was designed to be cleaved using UV light at 365 $\mathrm{nm}$. The release of 5-FU was controlled via monitoring the dose of the external stimulus. The conjugate polymer was more water soluble and had improved cell penetration ability (mean size less than $100 \mathrm{~nm}$ ) in comparison to LMWC, and produced hydrogel and DMSO-based gel. ${ }^{34}$

\section{Chitosan-PAsp}

Novel non-stoichiometric complex nanoparticles (NPs) based on CS and polyaspartic acid (PAsp) were formulated. All of the in vitro and in vivo analyses of 5-FUloaded NPs exhibited CS-controlled and slow release of 5FU, in contrast to 5-FU solution. Furthermore, the area under the curve (AUC) (an important parameter in pharmacokinetics) of the nano-based delivery systems was increased. $^{35}$

Folate-Tagged Chitosan Functionalized Gold NPs (CG-5FU-NPs and FCG-5-FU-NPs)

In a study by Akinyelu and Singh, 5-FU was loaded on gold NPs, with CS and folate-tagged CS. This nanostructure had great colloidal stability and $\mathrm{pH}$-dependent drug release behavior. Moreover, the kinetics of drug release was better fitted to a zero-order mechanism. Also, the anticancer effects of CG-5-FU-NPs and FCG-5-FU-NPs were superior to those of free 5-FU in the MCF-7 and HepG-2 cell lines. In addition, the study showed that FCG5-FU-NPs possessed higher cytotoxicity than CG-5-FUNPs in MCF-7 cells owing to the existence of folate receptors on these cells. ${ }^{36}$

Surface Molecularly Imprinted Polymer of ChitosanGrafted Poly(Methyl Methacrylate) (MIP-CS-g-PMMA)

Molecular imprinting has been used as smart polymers with particular recognition capacities for template molecules. $^{37,38}$ Zheng et al designed and prepared a novel oral-based colon-targeted 5-FU delivery system based on a molecularly imprinted method using CSPMMA as the matrix former for delivery of 5-FU through microspheres. The in vitro release mechanism was highly dependent on $\mathrm{pH}$ and time. 5-FU did not release at $\mathrm{pH} \mathrm{1,} \mathrm{slow} \mathrm{release} \mathrm{was} \mathrm{observed} \mathrm{at} \mathrm{pH} 6.8$ and fast release was achieved in the simulated colonic fluid ( $\mathrm{pH} \mathrm{7.4).}{ }^{39}$

\section{TMMIPs}

A type of $\mathrm{Fe}_{3} \mathrm{O}_{4}$-encapsulating carbon nanosphere with a shell of molecularly imprinted polymers, which are dually responsive to temperature and magnetism (TMMIPs), were produced by free radical polymerization. The synthesized nanospheres were used for controlled delivery of 5FU from aqueous media. These typical core-shell structures had a mean diameter of approximately $150 \mathrm{~nm}$, and the coating layer thickness was about $50 \mathrm{~nm}^{40}$

\section{PHBHHx}

Several kinds of microparticles (MPs) and NPs based on poly(3-hydroxybutyrate-co-3-hydroxyhexanoate)

(PHBHHx) were prepared by a double-emulsion solvent evaporation technique. They were utilized as the carrier of 5-FU. The characterization showed that the synthesized NPs and MPs had a spherical shape and sizes of approximately $160 \mathrm{~nm}$ for NPs and $3 \mu \mathrm{m}$ for MPs. This study revealed that cetyltrimethyl ammonium bromide (CTAB) as the emulsifier could enhance the drug loading content in both NPs and MPs. The in vitro drug release behavior was shown to be biphasic, with the initial burst release within 8 hours followed by a slow release rate throughout 24 hours. $^{41}$

\section{PLGA-Modified NPs}

In a study by Wang et al, a novel conjugate of poly(lacticco-glycolic acid) (PLGA) NPs was prepared. These NPs were conjugated with folic acid (FA), which is a suitable ligand for receptors on HT-29 cancer cells. They used 1,3diaminopropane as a cross-linker. This cross-linker was used to facilitate the conjugation of FA into PLGA-based 5-FU carriers. This fabrication had a great impact on specific targeted DDSs for antineoplastic agents. ${ }^{42}$

\section{BSA NPs (Protein-Based NPs)}

Protein-based NPs have attracted growing attention as new DDSs owing to their biodegradability and non-antigenicity. In a study conducted by Maghsoudi et al, 5-FUloaded BSA NPs were prepared by a phase separation technique. Taguchi's M16 design of experts was used in order to investigate the effect of process variables on the entrapment efficiency $(\% \mathrm{EE})$ of 5 -FU. It was found that $\mathrm{pH}$ had the most important effect on $\% \mathrm{EE}$ as the response parameter, and the amount of glutaraldehyde had the least important influence. This 5-FU-loaded BSA had a sustained release behavior, as it was capable of releasing drug based on zero kinetic release up to 20 hours. $^{43}$ 


\section{Calcium Phosphate Nanoparticles (CaP NPs)}

CaP NP have attracted great attention for anticancer drug deliveryowing to their biodegradability in nature, controlled release pattern and considerable loading efficiency. As a result, novel 5-FU-loaded CaP NPs (CaP-5-FU NPs) were prepared by a reverse micelle method. ${ }^{44}$

\section{PEG-PAMAM}

In a study conducted by Bhadra et al, the application of uncoated and PEGylated poly(amidoamine) (PAMAM) dendrimers as 5-FU delivery systems was examined. 4.0 G PAMAM dendrimers were synthesized via Michael addition and exhaustive amidation reactions. The core was ethylenediamine and the propagating agent was methylmethacrylate. In order to PEGylate the dendrimers, carboxymethyl methoxy polyethylene glycol (MPEG5000), which was previously activated by N-hydroxysuccinimide (NHS), was used. This reaction revealed enhancement of the drug loading content, controlled drug release behavior and reduced hemolytic toxicity. Also, PEGylation has been indicated to be advisable for fabrication of PAMAM dendrimers in order to reduce hematological disturbance and drug leakage. Moreover, this modification enhanced the drug loading capacity and was determined to be suitable for depot administration of 5-FU delivery systems. ${ }^{45}$

\section{PDEA-mPEG-PAMAM (PPD)}

As a new fabrication, a core-shell structure by $4.0 \mathrm{G}$ PAMAM, parallel poly(2-(N,N-diethylamino)ethyl methacrylate (PDEA) chains and $\mathrm{mPEG}$ chains (as the shell) was prepared. The PDEA chains were used owing to their $\mathrm{pH}-$ responsive behavior, and the PEG chains resulted in long circulation in bloodstream to target the tumor region. This polymer was used as the 5-FU carrier. As these nanocarriers were hydrophobic at $\mathrm{pH} 7.4$ and hydrophilic at $\mathrm{pH}$ 6.5 (owing to the pH-responsive PDEA chains), NP sizes, drug entrapment and release behavior were highly $\mathrm{pH}$ dependent. The release of 5-FU from this system at $\mathrm{pH}$ 7.4 was faster than at $\mathrm{pH}$ 6.5. Therefore, it is a promising system for antineoplastic drugs with high entrapment efficiency, $\mathrm{pH}$-responsive release and tumor-targeting capability. $^{46}$

\section{Poly(Lactic Acid) (PLA) and PLA-PEG}

5-FU-loaded PLA or PLA-PEG NPs were prepared via an emulsion solvent evaporation technique. The as-synthesized system could prolong 5-FU release but, surprisingly, did not reduce 5-FU cytotoxicity in the HepG2 cell line.
The researchers proved that all the pharmacokinetic parameters after a single oral administration in rats were improved. For instance, 5-FU bioavailability was enhanced four-fold in comparison to free 5-FU. Consequently, PLA and PLA-PEG nanoparticles have great potential as nanocarriers of 5-FU in oral delivery. ${ }^{47}$

\section{Magnetic Nanoparticles}

In a study by Hariharan et al, a series of 5-FU-loaded samarium ferrite $\left(\mathrm{SmFeO}_{3}\right)$ nanoparticles coated with poly(methylmethacrylate) was prepared. The drug was attached to the NPs by a host-guest complex with $\beta$ cyclodextrin $(\beta C D)$. The size was about $50 \mathrm{~nm}$, and the NPs showed a superparamagnetic behavior. In vitro cytotoxicity studies demonstrated the conservation of 5-FU potency and activity. ${ }^{48}$

\section{CEINs-PEI- $\beta$ CD-FA}

Carbon-encapsulated iron nanoparticles (CEINs) are used as theranostic probes. In a study conducted by Kasprzak et al, CEINs were chosen as the magnetic core of the DDS. In order to modify the surface of CEINs, branched polyethyleneimine (PEI) was utilized. FA was utilized as the targeting ligand, which is covalently attached to the CEINs, while $\beta C D$ was the host for 5-FU. The release pattern of 5-FU was investigated at various $\mathrm{pH}$ levels. By this examination, the highest release was seen at $\mathrm{pH} 9$ and the lowest at $\mathrm{pH}$ 4.7. So, this theranostic material has a controlled release behavior and could be a promising DDS in nanomedicine. ${ }^{49}$ In this kind of NP, the magnetic core was used for diagnosis, and by delivering 5-FU to the target site, this system was named a theranostic agent (having both therapeutic and diagnosis pathways) (Figure 3).

\section{Transferrin-Conjugated Liposomes}

Among the different vesicular systems, liposomes have gained interest as a site-specific delivery system, owing to their chemical and structural flexibility, along with their biocompatibility. ${ }^{50,51}$ In a study carried out by Soni et al, 5-FU-loaded transferrin-coupled liposomes were prepared by the cast film method. The in vivo results indicated a selective uptake of the nanocarrier from the endothelial cells of the brain. Approximately a 10-fold and 17-fold increase in the uptake of 5-FU to the brain after the entrapment by the liposomal system and transferrincoupled liposomes, respectively, was obtained,. ${ }^{52}$ 

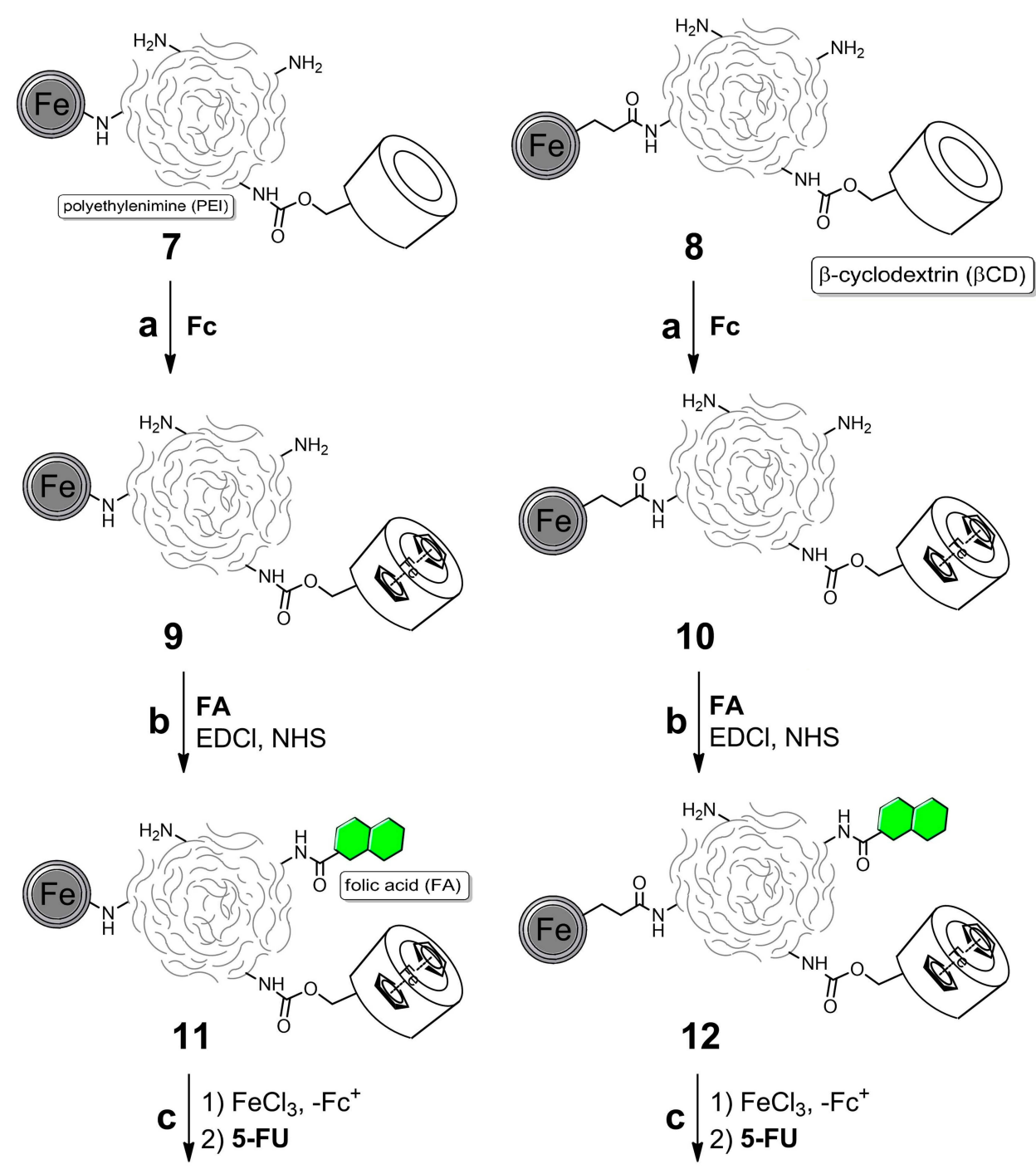

\section{2}

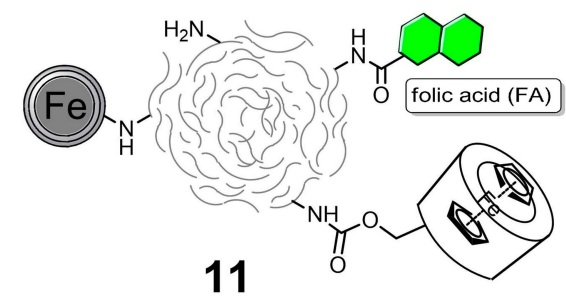

C $\begin{aligned} & \text { 1) } \mathrm{FeCl}_{3},-\mathrm{FC}^{+} \\ & \text {2) } 5 \text { - } \mathrm{FU}\end{aligned}$
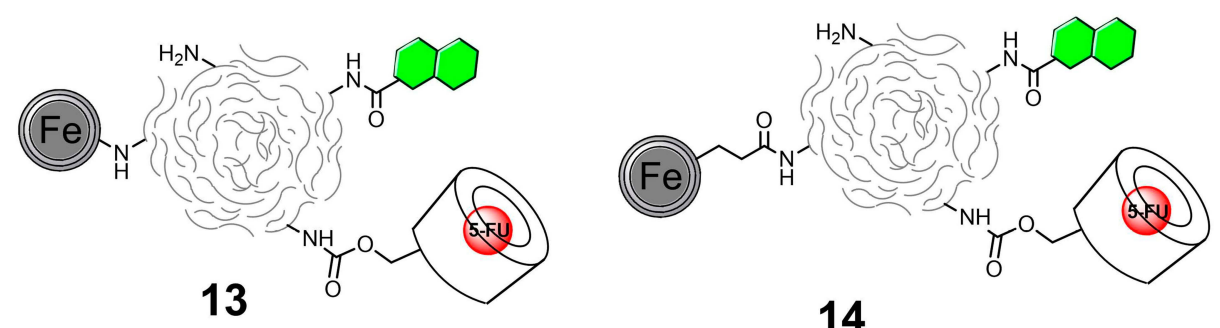

14

5-fluorouracil (5-FU) ferrocene (Fc)

Figure 3 Synthesis of CEINs-PEI-5-FU- $\beta C D-F A$ nanotheranostic agents.

Note: Copyright @ $\odot 2018$ Chemistry Select. Reproduce from Kasprzak A, Gunka K, Fronczak M, Bystrzejewski M, Poplawska M. Folic acid-navigated and $\beta$-cyclodextrin-decorated carbon-encapsulated iron nanoparticles as the nanotheranostic platform for controlled release of 5-fluorouracil. Chemistry Select. 2018;3 (38): $10821-10830 .^{49}$ 


\section{Folate-PEG-DSPE}

In a study conducted by Handali et al, optimization of a preparation of 5-FU-loaded phosphatidyl choline (PC) liposome was evaluated. FA was used as the targeting ligand for the surface receptors of HT-29, Caco-2 and MCF-7 cell lines. According to the results, the amount of $\mathrm{PC}$ was a significant variable in optimizing particle size and entrapment efficiency. Furthermore, in vitro cytotoxicity assays demonstrated that folate-conjugated liposomes had superior antitumor activity compared to the bare liposomes. ${ }^{53}$

\section{OFZG}

A phospholipase $\mathrm{A}_{2}$-sensitive amphiphilic prodrug, 1-Ooctadecyl-2-(5-fluorouracil)-N-acetyl-3-zidovudine-phosphorylglycerol (OFZG), was prepared and utilized as the nanoassembly during the injection of a mixture of Tween $80 / \mathrm{cholesterol} /$ OFZG $(0.1: 1: 2 \mathrm{~mol} / \mathrm{mol} / \mathrm{mol})$ in water. Secretory phospholipase $A_{2}$, which cleaves ester, is overexpressed in several types of cancer cells. The nanoassemblies exhibited improved anticancer activity compared to 5-FU in HCT116, HT-28 and COLO205 cell lines. The distribution of OFZG after intravenous administration was mainly in the kidney, spleen, lung and liver; it also exhibited rapid elimination. ${ }^{54}$

\section{Multi-Walled Carbon Nanotubes (MWCNTs)}

Carbon nanotubes (CNTs), as a developing type of nanomaterial, have been established as vehicles for gene and drug delivery. ${ }^{55}$ A group of researchers applied multiwalled carbon nanotubes (MWCNTs) to improve the cytotoxic and antitumoral effects of 5-FU. The drug was physically adsorbed on to MWCNTs, and the effect of drug both in vitro and in vivo was significantly improved. This study suggested that by using MWCNTs as carriers for antineoplastic drug delivery, not only would the efficacy be improved, but also the chances of resistance to cancer cells would be considerably diminished. ${ }^{56}$

\section{Silica Nanoparticles (EGF-HMSNs)}

Silica NPs provide unique properties as a drug delivery vehicle, including excellent stability and biocompatibility, along with a highly ordered structure of pores with uniform size.$^{57}$ In order to overwhelm ADR in CRC, a series of hollow mesoporous silica nanoparticles (HMSNs) conjugated with epidermal growth factor (EGF) was recruited as nanovehicles for 5-FU delivery to the SW480/ADR cell line, which is a CRC cell line with overexpression of EGF receptors. This type of nanocarrier was specifically internalized in the cells via receptor-mediated endocytosis and could successfully facilitate endosomal escape; by this method, ADR could be overcome in this cell line, resulting in cell death at the $\mathrm{S}$ phase of the cell cycle. ${ }^{18}$

\section{MAA-co-IA Hydrogel}

A new nanohydrogel based on methacrylic acid (MAA) and itaconic acid (IA), with ethylene glycol dimethacrylate (EGDMA) as a cross-linker, was synthesized for oral administration of 5-FU and leucovorin calcium (LV) to reach to the target site of the colon. This combinatorial DDS had a pH-responsive property to release the cargo at the $\mathrm{pH}$ of the colon ( $\mathrm{pH} 7.4$ ), but remain intact at acidic $\mathrm{pH}$. This property was due to the existence of carboxylic acid groups in the structure of this delivery system, which were able to ionize at higher $\mathrm{pH}$. The polymeric network is prone to swelling in this condition, and faster release of the loaded drug would be expected as a consequence of polymer swelling at $\mathrm{pH}$ 7.4. The release was better fitted to first order kinetics. ${ }^{58}$

\section{Non-Nano-Based Delivery Systems for 5- FU}

\section{Eudragit P-458IF/RSI00 Microspheres}

A colon-targeted MP, as an oral DDS, was prepared via an easy oil-in-water emulsification process. Eudragit $\mathrm{P}-4581 \mathrm{~F}$, which is a $\mathrm{pH}$-sensitive polymer, and Eudragit RS100, which prolongs drug release, were used in the formulation. This type of microsphere provided a colonspecific delivery system that permits release of the cargo at $\mathrm{pH} 7.4$, and prolonged release at the specific site would be expected owing to the presence of Eudragit RS100. This formulation would be a potential candidate for oral delivery of 5-FU. ${ }^{59}$

\section{Eudragit SI00 Electrospun Fibers}

Various series of 5-FU-loaded Eudragit S100 fibers were prepared by electrospinning to fabricate a $\mathrm{pH}$-sensitive oral delivery system. However, after characterization analyses, it was revealed that a very significant amount of 5FU was immediately released in stomach-mimicking media ( $\mathrm{pH} 1.0$ ) owing to the low molecular weight of 5$\mathrm{FU}$, which allows its diffusion through the fibers. Also, the loss of fiber integrity was another proposed reason for 5 FU escaping at the initial time of release. ${ }^{60}$

\section{Floating Bioadhesive Tablet (FBT)}

To target gastric cancer and diminish the dose and side effects, a series of FBTs of 5-FU was prepared. The tablets 
were made by a direct compression process. Hydroxyl propyl methyl cellulose (HPMC) was used to sustain the drug release and carbopol-971P, guar gum and sodium alginate were employed for their bioadhesive properties. This formulation could be successful as a gastric-targeted DDS $^{61}$

\section{PVC/PEG Polymeric Film}

In a study by Khalaf et al, a polymeric film based on polyvinyl chloride (PVC), PEG and starch cellulose acetate (SCA) was prepared. Trioctyltrimellitate, poly (MA-alt-NVP) (TOTM) was used as a plasticizer. It was suggested that a PVC/PEG/TOTM blend with a ratio of 10:90:5 had good mechanical properties. Moreover, the drug (5-FU) release pattern depended on the $\mathrm{pH}$ of the medium. In this regard, a sustained release pattern was observed, but a faster and higher release was detected at higher $\mathrm{pH}$. The cytotoxic assay indicated that release of 5-FU from the film had a sustained behavior in human liver cells. ${ }^{62}$

\section{Matrix Tablet with Pectin-Based Coat}

In order to obtain a colon-targeted 5-FU tablet, a microbially responsive pectin-based matrix tablet of 5-FU was prepared. Then, Eudragit S100 was used as a coating agent to restrict the release of 5-FU to the colon region. As a result, the systemic side effects were reduced and efficacy was improved. ${ }^{63}$

\section{Applying Drug Conjugates for 5-FU Delivery Poly(MA-alt-NVP)}

A water-soluble and alternating copolymer of poly(maleic anhydride-alt-N-vinyl pyrrolidonepoly(MA-alt-NVP) was synthesized via a charge transfer complex (CTC). The characterization of the copolymer was confirmed by attenuated total reflection-Fourier transform infrared, nuclear magnetic resonance, high-resolution Raman and X-ray diffraction spectroscopy. Then, conjugation of 5-FU to the copolymer was demonstrated by the aforementioned analyses. ${ }^{64}$ The results confirmed the successful copolymerization, and this copolymer could be used as a carrier for 5-FU to deliver this anticancer drug.

\section{Poly(Ethylene Oxide)-b-Poly(n-Butyl Methacrylate- co-4-Methyl-[7-(Methacryloyl)Oxyethyloxy] Coumarin)) (PEO-b-P(BMA-co-CMA))}

A series of block copolymers functionalized by coumarin was synthesized according to the atom transfer radical polymerization (ATRP) method. The drug-micelle conjugates were prepared by covalent bonding of 5-FU to the coumarin under UV radiation. Moreover, the drug release study was conducted under UV radiation (approximately $254 \mathrm{~nm}$ ), and the results demonstrated the controlled release of 5-FU from the conjugates. Therefore, a photoresponsive biocompatible 5-FU delivery system was prepared that would be a potential candidate for delivery of other antineoplastic agents. ${ }^{65}$

Table 2 Combinational Delivery System Containing 5-FU

\begin{tabular}{|l|l|l|l|}
\hline $\begin{array}{l}\text { Drug Used for } \\
\text { Co-Delivery }\end{array}$ & Type of Delivery System & Advantages/Improvement of Application & Reference \\
\hline Metformin & PFL/PPLL hydrogel & $\begin{array}{l}\text { Induces cell cycle arrest } \\
\text { Increases cytotoxicity of 5-FU }\end{array}$ & 69 \\
\hline Leucovorin Calcium & MAA-IA-EGDMA hydrogel & Synergizes the cytotoxicity activity of 5-FU & 78 \\
\hline As-miR-2I & PAMAM dendrimer & $\begin{array}{l}\text { Represses cell growth } \\
\text { Induces apoptosis } \\
\text { Increases cytotoxicity of 5-FU } \\
\text { Decreases adverse side reactions by targeting miR-2I overexpressing tumors }\end{array}$ & 70 \\
\hline LY294002 & PEGylated nanoliposome & $\begin{array}{l}\text { Enhances the sensitivity of tumor cells against 5-FU } \\
\text { Results in higher cytotoxicity }\end{array}$ & 72 \\
\hline CD-siRNA & LDH NPs & $\begin{array}{l}\text { Overwhelms the drug resistance } \\
\text { Improves cytotoxicity }\end{array}$ & 72 \\
\hline
\end{tabular}




\section{T22-GFP-H6-FdU}

In a study conducted by Céspedes et al, the active metabolite of 5-FU (floxuridine, FdU) was delivered to the CRC tumor. This cytotoxic agent was used to treat liver metastases of CRC. In this study, a nanoconjugate containing a fusion protein (the peptide T22 as a CXCR4 ligand, a green fluorescent protein and a histidine tail) was bound to FdU. This protein-based nanoconjugate selectively internalized and destroyed CXCR4 ${ }^{+}$CRC tumor cells and hence achieved a targeted DDS. ${ }^{66}$

\section{Combination Delivery of 5-FU}

Combinational chemotherapy is attracting more attention in comparison to single chemotherapy regimens. Combinatorial strategies in cancer treatment create a synergetic effect and, therefore, can enhances therapeutic efficiency. ${ }^{67}$ Using combinational therapeutics could be very effective owing to their enhanced curative efficacy in many diseases, such as malignant tumors. ${ }^{68}$ Table 2 presents some combination delivery systems discussed in this article.

\section{Metformin}

A nanohydrogel based on reactive aldehyde-functionalized 4-arm PEG (PFA) and four-arm PEG-b-poly(L-lysine) (PPLL) was prepared via Schiff's base reaction. This nanocarrier could simultaneously deliver metformin and 5-FU to colon 26 (C26) cell lines for improvement of antineoplastic activity and to overcome side effects. The combination treatment of 5-FU and metformin could induce cell cycle arrest and apoptosis, while subsequently increasing cytotoxicity both in vitro and in vivo. The release of both drugs was achieved in a controlled manner and $\mathrm{pH}$-dependent pattern. As a result, this injectable hydrogel could perform successfully in the treatment of CRC. ${ }^{69}$

\section{Leucovorin Calcium}

A novel hydrogel based on MAA and IA, with EGDMA as a cross-linker, was synthesized for oral administration of both 5-FU and LV to reach to the target site of the colon. LC administration with 5-FU was able to synergistically increase the cytotoxic activity and potency of 5-FU, as well as enhancing the therapeutic index. ${ }^{58}$

\section{As-miR-2I}

MicroRNAs (miRNAs) have been proven to be deregulated in various types of tumor cell. miR-21, as a pipeline in glioblastoma cells, can repress cell growth, induce cell apoptosis and lead to cell cycle arrest. Therefore, it can increase the therapeutic response of neoplastic drugs. In a study conducted by Ren et al, PAMAM dendrimer was used as a carrier for dual delivery of antisensemicroRNA21 (as-miR-21) oligonucleotide and 5-FU to accomplish the best result regarding cytotoxic efficacy. The MTT assay was performed in glioma cells (U251) and showed a dramatic increase in cytotoxicity. Therefore, this article suggested that co-delivery of asmiR-21 and 5-FU could have significant clinical outcomes in the cure of miR-21-overexpressing tumor cells. ${ }^{70}$

\section{Autophagy Inhibitor (LY294002)}

In this study, 5-FU and LY294002 were successfully loaded in PEGylated nanoliposomes to target esophageal squamous cell carcinoma (ESCC). This combinatorial drug delivery could lead to higher cytotoxic efficacy compared to single drug therapy. Also, the faster release of LY compared to 5-FU could result in the inhibition of autophagy, which enhanced the sensitivity of tumor cells to 5FU and induced a greater extent of apoptosis. ${ }^{71}$

\section{CD-siRNA}

Layered double hydroxide (LDH) NPs were employed as a vehicle for the co-delivery of 5-FU and Allstars Cell Death siRNA (CD-siRNA) to enhance cancer treatment. The LDH intercalated 5-FU into its interlayer space and also loaded siRNA on the external parts of LDH NPs. This type of NP has been indicated to be effective as an intracellular delivery system. Moreover, the combination of the antineoplastic drug 5-FU and CD-siRNA could significantly improve cytotoxicity in HCT-116, U2OS and MCF-7 cell lines, in comparison to single therapy, owing to the simultaneous mitochondrial damage process. Consequently, the approach of co-delivery of siRNA and 5-FU by LDH NPs could be promising in overwhelming the drug resistance and improving cancer treatment. ${ }^{72}$

\section{Future Outlook and Conclusion}

The application of novel DDSs in the field of pharmaceutical sciences has revolutionized healthcare systems, because carrier-based advanced DDSs can significantly reduce the adverse effects of drugs and improve the drug efficacy. In this review, nano-based delivery systems of 5-FU were highlighted. This type of DDS has the potential to enhance the physicochemical aspects of drug characteristics and can effectively target 5-FU to the cancerous region. Moreover, today the field of nanomedicine is taking the lead for personalized 
medicine. ${ }^{73}$ So, it is important to use this kind of novel technology to carry the drugs. All types of nanocarrier, including CNTs, liposomes, polymeric NPs, silica NPs, dendrimers and micelles, have advantages over conventional delivery systems and, in most cases, outweigh the disadvantages.

Furthermore, combining therapeutic agents with 5-FU to enhance therapeutic efficacy is another beneficial ability of novel delivery systems for the treatment of CRC. This strategy can also enhance the sensitivity of tumor cells to 5-FU and overcome drug resistance.

Another strategy is the application of stimuli-responsive nanocarriers to achieve passive targeting delivery. The physical/chemical and internal/external stimuli provide precise control of 5-FU release and, therefore, can reduce severe side effects following premature release. ${ }^{4}$

We believe that in the future, nanomedicine-based codelivery DDSs will be more promising than conventional drugs to cure cancers, but more attempts must be completed to enhance the pharmacokinetic aspects of this type of DDS and successfully translating to the clinic.

\section{Abbreviations}

5-FU, 5-fluorouracil; CRC, colorectal cancer; GI, gastrointestinal; DDS, drug delivery system; FdUMP, fluorodeoxyuridine monophosphate; FdUTP, fluorodeoxyuridine triphosphate; FUTP, fluorouridine triphosphate; DHFU, dihydrofluorouracil; DPD, dihydropyrimidine dehydrogenase; ADR, acquired drug resistance; SLN, solid lipid nanoparticle; CS, chitosan; CS-g-PCL, chitosan-graft-poly $(\varepsilon-$ caprolactone) micelle; LMWC, low molecular weight chitosan; AUC, area under the curve; NP, nanoparticle; MIP-CSg-PMMA, surface molecularly imprinted polymer of chitosan-grafted poly(methyl methacrylate); TMMIP, temperature and magnetic responsive molecularly imprinted polymer; PHBHHx, poly(3-hydroxybutyrate-co-3-hydroxyhexanoate); MP, microparticle; CTAB, cetyltrimethyl ammonium bromide; PLGA, poly(lactic-co-glycolic acid); FA, folic acid; BSA, bovine serum albumin; EE, entrapment efficiency; PEG, polyethylene glycol; PAMAM, poly(amidoamine); NHS, N-hydroxysuccinimide; mPEG, methoxy polyethylene glycol; PDEA, poly(2-(N,N-diethylamino) ethyl methacrylate; PLA, poly(lactic acid); $\beta \mathrm{CD}, \beta$-cyclodextrin; CEIN, carbon-encapsulated iron nanoparticle; PEI, polyethyleneimine; PC, phosphatidyl choline; OFZG, 1-Ooctadecyl-2-(5-fluorouracil)-N-acetyl-3-zidovudine-phosphorylglycerol; CNT, carbon nanotube; MWCNT, multiwalled carbon nanotube; HMSN, hollow mesoporous silica nanoparticle; EGF, epidermal growth factor; MAA, methacrylic acid; IA, itaconic acid; EGDMA, ethylene glycol dimethacrylate; LV, leucovorin calcium; FBT, floating bioadhesive tablet; HPMC, hydroxyl propyl methyl cellulose; PVC, polyvinyl chloride; SCA, starch cellulose acetate; TOTM, trioctyltrimellitate; poly(MA-alt-NVP), poly(maleic anhydride-alt-N-vinyl pyrrolidone); CTC, charge transfer complex; PEO-b-P(BMA-co-CMA), poly(ethylene oxide)$b$-poly(n-butyl methacrylate-co-4-methyl-[7-(methacryloyl) oxyethyloxy]coumarin)); ATRP, atom transfer radical polymerization; as-miR-21, antisense-microRNA21; ESCC, esophageal squamous cell carcinoma; CD-siRNA, Allstars Cell Death siRNA; LDH, layered double hydroxide.

\section{Acknowledgment}

The authors would like to thank the Research Council of Shiraz University of Medical Sciences for supporting this project under grant no. 99-01-106-22797. This article is part of Elaheh Entezar-Almahdi's $\mathrm{PhD}$ thesis.

\section{Disclosure}

The authors declare no conflicts of interest in this article.

\section{References}

1. Krishnaiah Y, Satyanarayana V, Kumar BD, Karthikeyan R. In vitro drug release studies on guar gum-based colon targeted oral drug delivery systems of 5-fluorouracil. Eur J Pharm Sci. 2002;16 (3):185-192. doi:10.1016/S0928-0987(02)00081-7

2. Lamprecht A, Yamamoto H, Takeuchi H, Kawashima Y. Observations in simultaneous microencapsulation of 5-fluorouracil and leucovorin for combined pH-dependent release. Eur J Pharm Biopharm. 2005;59 (2):367-371. doi:10.1016/j.ejpb.2004.09.005

3. Hosseini M, Farjadian F, Makhlouf ASH. Smart Stimuli-Responsive Nano-Sized Hosts for Drug Delivery. Industrial Applications for Intelligent Polymers and Coatings. Cham: Springer; 2016:1-26.

4. Farjadian F, Roointan A, Mohammadi-Samani S, Hosseini M. Mesoporous silica nanoparticles: synthesis, pharmaceutical applications, biodistribution, and biosafety assessment. Chem Eng. 2019;359:684-705. doi:10.1016/j.cej.2018.11.156

5. Farjadian F, Ghasemi A, Gohari O, Roointan A, Karimi M, Hamblin MR. Nanopharmaceuticals and nanomedicines currently on the market: challenges and opportunities. Nanomedicine. 2019;14(1):93-126. doi:10.2217/nnm-2018-0120

6. Farjadian F, Moghoofei M, Mirkiani S, et al. Bacterial components as naturally inspired nano-carriers for drug/gene delivery and immunization: set the bugs to work? Biotechnol Adv. 2018;36(4):968-985. doi:10.1016/j.biotechadv.2018.02.016

7. Roointan A, Farzanfar J, Mohammadi-Samani S, Behzad-Behbahani A, Farjadian F. Smart pH responsive drug delivery system based on poly (HEMA-co-DMAEMA) nanohydrogel. Int J Pharm. 2018;552(12):301-311. doi:10.1016/j.ijpharm.2018.10.001

8. Farjadian F, Rezaeifard S, Naeimi M, et al. Temperature and $\mathrm{pH}-$ responsive nano-hydrogel drug delivery system based on lysine-modified poly (vinylcaprolactam). Int J Nanomedicine. 2019;14:6901. doi:10.2147/IJN.S214467

9. Longley DB, Harkin DP, Johnston PG. 5-fluorouracil: mechanisms of action and clinical strategies. Nat Rev Cancer. 2003;3(5):330. doi: $10.1038 / \mathrm{nrc} 1074$ 
10. Sun X, Liu C, Omer A, et al. $\mathrm{pH}$-sensitive $\mathrm{ZnO} /$ carboxymethyl cellulose/chitosan bio-nanocomposite beads for colon-specific release of 5-fluorouracil. Int J Biol Macromol. 2019;128:468-479. doi:10. 1016/j.ijbiomac.2019.01.140

11. Krishnaiah Y, Satyanarayana V, Kumar BD, Karthikeyan R, Bhaskar P. In vivo pharmacokinetics in human volunteers: oral administered guar gum-based colon-targeted 5-fluorouracil tablets. Eur J Pharm Sci. 2003;19(5):355-362. doi:10.1016/S0928-0987(03)00139-8

12. Burki TK. TAS-102 in metastatic colorectal cancer. Lancet Oncol. 2018;19(1):e18. doi:10.1016/S1470-2045(17)30927-0

13. Fournier E, Passirani C, Colin N, Breton P, Sagodira S, Benoit J-P. Development of novel 5-FU-loaded poly (methylidene malonate 2.1. 2)-based microspheres for the treatment of brain cancers. Eur J Pharm Biopharm. 2004;57(2):189-197. doi:10.1016/S0939-6411(03)00146-2

14. Minko T, Dharap S, Pakunlu R, Wang Y. Molecular targeting of drug delivery systems to cancer. Curr Drug Targets. 2004;5(4):389-406. doi: $10.2174 / 1389450043345443$

15. Minko T. Drug targeting to the colon with lectins and neoglycoconjugates. Adv Drug Deliv Rev. 2004;56(4):491-509. doi:10.1016/j.addr.2003. 10.017

16. Wohlhueter RM, McIvor RS, Plagemann PG. Facilitated transport of uracil and 5-fluorouracil, and permeation of orotic acid into cultured mammalian cells. J Cell Physiol. 1980;104(3):309-319. doi:10.1002/ jcp. 1041040305

17. Okumura K, Shiomi H, Mekata E, et al. Correlation between chemosensitivity and mRNA expression level of 5-fluorouracil-related metabolic enzymes during liver metastasis of colorectal cancer. Oncol Rep. 2006;15(4):875-882.

18. Chen L, She X, Wang T, et al. Overcoming acquired drug resistance in colorectal cancer cells by targeted delivery of 5-FU with EGF grafted hollow mesoporous silica nanoparticles. Nanoscale. 2015;7 (33):14080-14092. doi:10.1039/C5NR03527A

19. Ma Z, Ma R, Wang X, Gao J, Zheng Y, Sun Z. Enzyme and PH responsive 5-flurouracil (5-FU) loaded hydrogels based on olsalazine derivatives for colon-specific drug delivery. Eur Polym J. 2019;118:64-70. doi:10.1016/j.eurpolymj.2019.05.017

20. Kaur V, Goyal AK, Ghosh G, Si SC, Rath G. Development and characterization of pellets for targeted delivery of 5-fluorouracil and phytic acid for treatment of colon cancer in wistar rat. Heliyon. 2020;6(1):e03125. doi:10.1016/j.heliyon.2019.e03125

21. Hu X, Jing X. Biodegradable amphiphilic polymer-drug conjugate micelles. Expert Opin Drug Deliv. 2009;6(10):1079-1090. doi:10.1517/17425240903158917

22. Hu X, Li J, Lin W, Huang Y, Jing X, Xie Z. Paclitaxel prodrug nanoparticles combining chemical conjugation and physical entrapment for enhanced antitumor efficacy. RSC Adv. 2014;4(72):3840538411. doi:10.1039/C4RA06270A

23. Ogawara K-I, Yoshizawa Y, Un K, Araki T, Kimura T, Higaki K. Nanoparticle-based passive drug targeting to tumors: considerations and implications for optimization. Biol Pharm Bull. 2013;36(5):698702. doi:10.1248/bpb.b13-00015

24. Jabr-Milane L, van Vlerken L, Devalapally H, et al. Multi-functional nanocarriers for targeted delivery of drugs and genes. J Control Release. 2008;130(2):121-128. doi:10.1016/j.jconrel.2008.04.016

25. Kircheis R, Wightman L, Schreiber A, et al. Polyethylenimine/DNA complexes shielded by transferrin target gene expression to tumors after systemic application. Gene Ther. 2001;8(1):28-40. doi:10.1038/sj. gt.3301351

26. Sheikhsaran F, Sadeghpour H, Khalvati B, Entezar-Almahdi E, Dehshahri A. Tetraiodothyroacetic acid-conjugated polyethylenimine for integrin receptor mediated delivery of the plasmid encoding IL-12 gene. Colloids Surf B Biointerfaces. 2017;150:426-436. doi:10.1016/ j.colsurfb.2016.11.008
27. Sadeghpour H, Khalvati B, Entezar-Almahdi E, et al. Double domain polyethylenimine-based nanoparticles for integrin receptor mediated delivery of plasmid DNA. Sci Rep. 2018;8(1):1-12. doi:10.1038/ s41598-018-25277-z

28. Benns JM, Mahato RI, Kim SW. Optimization of factors influencing the transfection efficiency of folate-PEG-folate-graft-polyethylenimine. J Control Release. 2002;79(1-3):255-269. doi:10.1016/ S0168-3659(01)00513-2

29. Mohammadi-Samani S, Zojaji S, Entezar-Almahdi E. Piroxicam loaded solid lipid nanoparticles for topical delivery: preparation, characterization and in vitro permeation assessment. J Drug Deliv Sci Technol. 2018;47:427-433. doi:10.1016/j.jddst.2018.07.015

30. Ghasemiyeh P, Mohammadi-Samani S. Solid lipid nanoparticles and nanostructured lipid carriers as novel drug delivery systems: applications, advantages and disadvantages. Res Pharm Sci. 2018;13(4):288. doi:10.4103/1735-5362.235156

31. Mohammadi-Samani S, Salehi H, Entezar-Almahdi E, Masjedi M. Preparation and characterization of sumatriptan loaded solid lipid nanoparticles for transdermal delivery. J Drug Deliv Sci Technol. 2020;57:101719. doi:10.1016/j.jddst.2020.101719

32. Khallaf RA, Salem HF, Abdelbary A. 5-Fluorouracil shell-enriched solid lipid nanoparticles (SLN) for effective skin carcinoma treatment. Drug Deliv. 2016;23(9):3452-3460. doi:10.1080/ 10717544.2016.1194498

33. Gu C, Le V, Lang M, Liu J. Preparation of polysaccharide derivates chitosan-graft-poly ( $\varepsilon$-caprolactone) amphiphilic copolymer micelles for 5-fluorouracil drug delivery. Colloids Surf B Biointerfaces. 2014;116:745-750. doi:10.1016/j.colsurfb.2014.01.026

34. Horo H, Das S, Mandal B, Kundu LM. Development of a photoresponsive chitosan conjugated prodrug nano-carrier for controlled delivery of antitumor drug 5-fluorouracil. Int J Biol Macromol. 2019;121:1070-1076. doi:10.1016/j.ijbiomac.2018.10.095

35. Zheng Y, Yang W, Wang C, et al. Nanoparticles based on the complex of chitosan and polyaspartic acid sodium salt: preparation, characterization and the use for 5-fluorouracil delivery. Eur $J$ Pharm Biopharm. 2007;67(3):621-631. doi:10.1016/j. ejpb.2007.04.007

36. Akinyelu J, Singh M. Folate-tagged chitosan-functionalized gold nanoparticles for enhanced delivery of 5-fluorouracil to cancer cells. Appl Nanosci. 2018;1-11.

37. Yang -H-H, Zhang S-Q, Tan F, Zhuang Z-X, Wang X-R. Surface molecularly imprinted nanowires for biorecognition. J Am Chem Soc. 2005;127(5):1378-1379. doi:10.1021/ja0467622

38. Matsui J, Higashi M, Takeuchi T. Molecularly imprinted polymer as 9-ethyladenine receptor having a porphyrin-based recognition center. J Am Chem Soc. 2000;122(21):5218-5219.

39. Zheng X-F, Lian Q, Yang H, Wang X. Surface molecularly imprinted polymer of chitosan grafted poly(methyl methacrylate) for 5-fluorouracil and controlled release. Sci Rep. 2016;6(1):21409. doi:10.1038/srep21409

40. Li L, Chen L, Zhang H, Yang Y, Liu X, Chen Y. Temperature and magnetism bi-responsive molecularly imprinted polymers: preparation, adsorption mechanism and properties as drug delivery system for sustained release of 5-fluorouracil. Mater Sci Eng C. 2016;61:158-168. doi:10.1016/j.msec.2015.12.027

41. Lu XY, Zhang Y, Wang L. Preparation and in vitro drug-release behavior of 5-fluorouracil-loaded poly (hydroxybutyrate-co-hydroxyhexanoate) nanoparticles and microparticles. J Appl Polym Sci. 2010;116(5):2944-2950.

42. Wang Y, Li P, Chen L, Gao W, Zeng F, Kong LX. Targeted delivery of 5-fluorouracil to HT-29 cells using high efficient folic acid-conjugated nanoparticles. Drug Deliv. 2015;22(2):191-198. doi:10.3109/ 10717544.2013 .875603 
43. Maghsoudi A, Shojaosadati SA, Farahani EV. 5-Fluorouracil-loaded BSA nanoparticles: formulation optimization and in vitro release study. AAPS PharmSciTech. 2008;9(4):1092-1096. doi:10.1208/ s12249-008-9146-5

44. Mohiyuddin S, Naqvi S, Packirisamy G. Enhanced antineoplastic/ therapeutic efficacy using 5-fluorouracil-loaded calcium phosphate nanoparticles. Beilstein J Nanotechnol. 2018;9(1):2499-2515. doi:10.3762/bjnano.9.233

45. Bhadra D, Bhadra S, Jain S, Jain N. A PEGylated dendritic nanoparticulate carrier of fluorouracil. Int J Pharm. 2003;257(1-2):111124. doi:10.1016/S0378-5173(03)00132-7

46. Jin Y, Ren X, Wang W, et al. A 5-fluorouracil-loaded $\mathrm{pH}$-responsive dendrimer nanocarrier for tumor targeting. Int $J$ Pharm. 2011;420 (2):378-384. doi:10.1016/j.ijpharm.2011.08.053

47. Mattos A, Altmeyer C, Tominaga TT, Khalil NM, Mainardes RM. Polymeric nanoparticles for oral delivery of 5-fluorouracil: formulation optimization, cytotoxicity assay and pre-clinical pharmacokinetics study. Eur J Pharm Sci. 2016;84:83-91. doi:10.1016/j. ejps.2016.01.012

48. Hariharan MS, Sivaraj R, Ponsubha S, Jagadeesh R, Enoch IVMV. 5Fluorouracil-loaded $\beta$-cyclodextrin-carrying polymeric poly(methylmethacrylate)-coated samarium ferrite nanoparticles and their anticancer activity. J Mater Sci. 2018.

49. Kasprzak A, Gunka K, Fronczak M, Bystrzejewski M, Poplawska M. Folic acid-navigated and $\beta$-cyclodextrin-decorated carbon-encapsulated iron nanoparticles as the nanotheranostic platform for controlled release of 5-fluorouracil. ChemistrySelect. 2018;3(38):10821-10830. doi:10.1002/slct.201802318

50. Simoes S, Fonseca C, de Lima MP, Düzgünes N. Ph-Sensitive Liposomes: From Biophysics to Therapeutic Applications. Trigger Sensitive Assemblies and Particulates. London: Citus Books; 2005.

51. Storm G, Koppenhagen F, Heeremans A, Vingerhoeds M, Woodle MC, Crommelin DJ. Novel developments in liposomal delivery of peptides and proteins. J Control Release. 1995;36(1-2):19-24. doi:10.1016/0168-3659(95)00047-C

52. Soni V, Kohli D, Jain S. Transferrin-conjugated liposomal system for improved delivery of 5-fluorouracil to brain. J Drug Target. 2008;16 (1):73-78. doi:10.1080/10611860701725381

53. Handali S, Moghimipour E, Kouchak M, et al. New folate receptor targeted nano liposomes for delivery of 5-fluorouracil to cancer cells: strong implication for enhanced potency and safety. Life Sci. 2019;227:39-50. doi:10.1016/j.lfs.2019.04.030

54. Jin Y, Yang F, Du L. Nanoassemblies containing a fluorouracil/ zidovudine glyceryl prodrug with phospholipase A2-triggered drug release for cancer treatment. Colloids Surf $B$ Biointerfaces. 2013;112:421-428. doi:10.1016/j.colsurfb.2013.08.021

55. Entezar-Almahdi E, Morowvat MH. Pharmacokinetic aspects of carbon nanotubes: improving outcomes of functionalization. Curr Nanosci. 2019;15(5):454-459. doi:10.2174/1573413715666 181204113525

56. González-Lavado E, Valdivia L, García-Castaño A, et al. Multiwalled carbon nanotubes complement the anti-tumoral effect of 5fluorouracil. Oncotarget. 2019;10(21):2022.

57. Ohulchanskyy TY, Roy I, Goswami LN, et al. Organically modified silica nanoparticles with covalently incorporated photosensitizer for photodynamic therapy of cancer. Nano Lett. 2007;7(9):2835-2842. doi:10.1021/n10714637

58. Abdullah O, Usman Minhas M, Ahmad M, Ahmad S, Ahmad A. Synthesis of hydrogels for combinatorial delivery of 5-fluorouracil and leucovorin calcium in colon cancer: optimization, in vitro characterization and its toxicological evaluation. Polym Bull. 2018.
59. Lamprecht A, Yamamoto H, Takeuchi H, Kawashima Y. Microsphere design for the colonic delivery of 5-fluorouracil. J Control Release. 2003;90(3):313-322. doi:10.1016/S0168-3659(03)00195-0

60. Illangakoon UE, Yu D-G, Ahmad BS, Chatterton NP, Williams GR. 5-fluorouracil loaded eudragit fibers prepared by electrospinning. Int J Pharm. 2015;495(2):895-902. doi:10.1016/j.ijpharm.2015.09.044

61. Panigrahy RN, Panda SK, Veerareddy PR. Formulation and evaluation of stomach-specific novel gastro-retentive formulations of 5fluorouracil for targeting gastric cancer. Int J Pharm Sci Rev Res. 2018;9(9):3795-3803.

62. Khalaf AI, El Nashar DE, Helaly FM, Soliman A. Evaluation of controlled release PVC/PEG polymeric films containing 5-fluorouracil for long-term antitumor. Polym Bull. 2018.

63. Dev RK, Bali V, Pathak K. Novel microbially triggered colon specific delivery system of 5-fluorouracil: statistical optimization, in vitro, in vivo, cytotoxic and stability assessment. Int J Pharm. 2011;411(12):142-151. doi:10.1016/j.ijpharm.2011.03.057

64. Parvizikhosroshahi S, Can HK. Synthesis and characterization of novel polymer-drug conjugate based on the anhydride containing copolymer as a potential method for drug carrier. J Macromol Sci A. 2018;55(2):192-204. doi:10.1080/10601325.2017.1410064

65. Jin Q, Mitschang F, Agarwal S. Biocompatible drug delivery system for photo-triggered controlled release of 5-fluorouracil. Biomacromolecules. 2011;12(10):3684-3691. doi:10.1021/bm20 09125

66. Céspedes MV, Unzueta U, Aviñó A, et al. Selective depletion of metastatic stem cells as therapy for human colorectal cancer. $E M B O$ Mol Med. 2018;10(10). doi:10.15252/emmm.201708772.

67. Liu H, Zang C, Emde A, et al. Anti-tumor effect of honokiol alone and in combination with other anti-cancer agents in breast cancer. Eur J Pharmacol. 2008;591(1-3):43-51. doi:10.1016/j.ejphar. 2008.06.026

68. Kaplan SA, McConnell JD, Roehrborn CG, et al. Combination therapy with doxazosin and finasteride for benign prostatic hyperplasia in patients with lower urinary tract symptoms and a baseline total prostate volume of $25 \mathrm{~mL}$ or greater. J Urol. 2006;175(1):217-220. doi:10.1016/S0022-5347(05)00041-8

69. Wu X, He C, Wu Y, Chen X. Synergistic therapeutic effects of Schiff's base cross-linked injectable hydrogels for local co-delivery of metformin and 5-fluorouracil in a mouse colon carcinoma model. Biomaterials. 2016;75:148-162. doi:10.1016/j.biomaterials.2015. 10.016

70. Ren Y, Kang C-S, Yuan X-B, et al. Co-delivery of as-miR-21 and 5FU by poly (amidoamine) dendrimer attenuates human glioma cell growth in vitro. J Biomater Sci Polym Ed. 2010;21(3):303-314. doi:10.1163/156856209X415828

71. Feng Y, Gao Y, Wang D, Xu Z, Sun W, Ren P. Autophagy inhibitor (LY294002) and 5-fluorouracil (5-FU) combination-based nanoliposome for enhanced efficacy against esophageal squamous cell carcinoma. Nanoscale Res Lett. 2018;13(1):325. doi:10.1186/s11671-0182716-x

72. Li L, Gu W, Chen J, Chen W, Xu ZP. Co-delivery of siRNAs and anti-cancer drugs using layered double hydroxide nanoparticles. Biomaterials. 2014;35(10):3331-3339. doi:10.1016/j.biomaterials. 2013.12.095

73. Drbohlavova J, Chomoucka J, Adam V, et al. Nanocarriers for anticancer drugs-new trends in nanomedicine. Curr Drug Metab. 2013;14 (5):547-564. doi:10.2174/1389200211314050005 


\section{Publish your work in this journal}

The International Journal of Nanomedicine is an international, peerreviewed journal focusing on the application of nanotechnology in diagnostics, therapeutics, and drug delivery systems throughout the biomedical field. This journal is indexed on PubMed Central, MedLine, CAS, SciSearch ${ }^{\circledR}$, Current Contents ${ }^{\mathbb{B}} /$ Clinical Medicine, $^{2}$
Journal Citation Reports/Science Edition, EMBase, Scopus and the Elsevier Bibliographic databases. The manuscript management system is completely online and includes a very quick and fair peer-review system, which is all easy to use. Visit http://www.dovepress.com/ testimonials.php to read real quotes from published authors. 